\title{
NUMERICAL INVESTAGATION OF THE MECHANISMS OF DESICCATION CRACKING IN FINE-GRAINED SOILS
}

\author{
Y. Gui, G. Zhao, N. Khalili
}

School of Civil and Environmental Engineering, The University of New South Wales. Kensington, Sydney, Australia, 2052 (y.gui@unsw.edu.au)

\begin{abstract}
Numerical simulations for desiccation shrinking and cracking in fine-grained soil, based on Distinct Lattice Spring Model by using a new developed micro mechanism, are performed. Negative pore water pressure and air entry value during drying are accountted for through a new introduced suction force constitutive law which describes the mechanical process of desiccation. Drying cracking of heterogeneous soil can be expressed by failure of the suction force at different time and different position where pore draining to dry is happening. The parameters obtained from free drying simulation are used to model the process of constrained drying. Comparisons are made between the simulation results and experimental data highlighting the capability of the model. A good agreement between simulation and exprimental presentation has been reached. In addition, the influences of random ratio and particle size are discussed in this paper as well.
\end{abstract}

Keywords: Desiccation cracking, Distinct Lattice Spring Model, Air-entry, Crack pattern.

\section{INTRODUCTION}

Desiccation cracking is a common phenomenon in geotechnical engineering. Cracking induces soil structure, forming the boundaries between incipient soil aggregates and major transmission pathways for water and chemicals [5]. During desiccation, soil water removes from soil and the soil volume decreases. This issue is one of the main concerns in the design and construction of landfills, especially in arid regions, as compressibility increases significantly whereas the rate of consolidation decreases [10, 17]. Desiccation cracking is an invisible disaster in industry: crack in fine-grained mining waste or tailing not only changes the permeability of tailings, but also induces environmental consequences because of potential pollutant generation on infiltration (heavy metal pollution) [18]; cracking also increases the accessibility of oxygen, which enables oxidation of the tailing and the potential generations of acid mine drainage; in dam, embankment and slope engineering, desiccation cracks are a possible precursor for inception of failure surface at the top of dams, embankments and slopes, stated by [16]; due to desiccation cracking, it leads to drastic increase of permeability of the buffers, then cracks become pathway of contaminated leakage [4]. However, cracking in agriculture production is an important guarantee of crop. The effects on crop production 
embodies in two aspects: on one hand, cracks constitute the macro-pores which are needed for the drainage of excess water; on the other hand, cracks constitute the pores that are essential for the flow of water and air to plants [22]. Therefore, the research of desiccation crack in soil is an issue of importance and significance for researchers.

Cracks are likely to occur if soil is not free from shrinkage and if tensile stresses generated in the soil reach its tensile strength. Shrinkage constraints are from frictional traction, displacement boundary conditions, eign-stress concentration, soil texture and soil structures [11,16]. Frictional traction, displacement boundary condition and eign-stress concentration has been studied [16]. This paper will focus on soil intrinsic factor (i.e. soil texture and structures). As the response to desiccation, suction and tension increase along with the decrease of water content. When suction reaches a critical state (i.e. pore air entry value), water-air interface (meniscus) invades into pores, and then cracks would be formed on the surface, resulting in the decrease of some tension energy [21]. Commonly accepted criterion is to consider that a desiccation crack is initiated when the tensile stress generated during drying exceeds the tensile strength of the material [9]. A lot of works in this area have been performed with emphasis on desiccation of clay soils $[11,16]$, chemical shrinkage of clay soils [7] and shrinkage cracking of cemented geomaterials [12]. Based on these results, some laboratory tests of desiccation cracking and preliminary results of a simplified analytical method developed for the prediction of crack formation were presented [8]. However, the tensile strength of the material is a function of the original flaw distribution, the soil-water suction and the water content within the material [22]. Besides, if the effective stress principle is adopted to explain the desiccation shrinking and cracking, the compressive stress instead of tension will be obtained. That is the strange point when effective stress principle is used. Thus, despite these advances, a general approach to predicting crack development is still not available.

In order to explain it, microscopic analysis method is introduced. Basically, soil grains are connected with each other by matric suction (water bond). Due to the heterogeneity of water evaporation, some pores are drained first compared with their neighbouring pores. Due to the drying, the suction is increasing. However, when the pore totally drained, the tensile stress in this pore will vanish immediately then this pore expands and propagates by the tensile force from neighbouring undrained pores. Recently, with the advancement of computer technology, more and more numerical methods have been adopted as effective tools to simulate and reproduce desiccation shrinkage and cracking $[3,15,20]$. Nevertheless, the crack prediction (onset and propagation) has not been cleared yet. Peron [15] proposed a DEM model trying to explain desiccation shrinkage and cracking by decreasing particle size with time, which is not the case of desiccation. In this paper, Distinct Lattice Spring Model (DLSM) is used and soil crack initiation and propagation are modelled based on the heterogeneity and suction force constitutive model, which explains the incentives of desiccation crack from microscopic perspective. 


\section{DISTINCT LATTICE SPRING MODEL ON DESICCATION CRACKING}

\subsection{The Distinct Lattice Spring Model (DLSM)}

Lattice models $[1,13]$ represent material by a system of discrete units (e.g. particles) interacting via springs, or, more generally, rheological elements. These discrete units are much coarser than the true atomic ones and may represent larger volumes of heterogeneities such as grains or clusters of grains. Due to their discrete nature, lattice models are known to be more suitable for complex fracturing simulation. For example, lattice models have been successfully applied to investigate the spatial cooperative effects of crack formation and heterogeneities in elastic-plastic [2] and elastic-brittle [14] systems. However, for lattice models composed of normal springs transmitting central forces only, it is known that the modelled Poisson's ratio approaches, in the limit of an infinite number of particles, a fixed value e.g. 1/4 in three-dimensional cases. Recently, an alternative 3D lattice spring model, Distinct Lattice Spring Model (DLSM) [24-25] has been proposed to overcome the restriction on the Poisson's ratio while preserving the rotational invariance. In DLSM, materials are discretized into mass particles linked through distributed bonds (see Figure 1(a)). Whenever two particles are detected in contact, they are linked together through bonds between their centre points. Based on Cauchy-Born rules and the hyper-elastic theory, the relationship between the micromechanical parameters and the macro-material constants can be obtained as follows:

$$
\begin{gathered}
k_{n}=\frac{3 E}{\alpha^{3 D}(1-2 v)} . \\
k_{s}=\frac{3(1-4 v) E}{\alpha^{3 D}(1+v)(1-2 v)} .
\end{gathered}
$$

where $k_{n}$ is the spring normal stiffness, $k_{s}$ is the shear stiffness, $E$ is the Young's modulus, $v$ is the Poisson's ratio and $\alpha^{3 D}$ is the microstructure geometry coefficient, which is obtained from

$$
\alpha^{3 D}=\frac{\sum l_{i}^{2}}{V}
$$

in which $l_{i}$ is the original length of the $i^{\text {th }}$ bond and $V$ is the volume of the geometry model. The particles and springs comprise a whole system, which represents the material. For this system, the equation of motion is expressed as

$$
[\mathbf{K}] \mathbf{u}+[\mathbf{C}] \dot{\mathbf{u}}+[\mathbf{M}] \ddot{\mathbf{u}}=\mathbf{F}(t)
$$

where $\mathbf{u}$ represents the particle displacement vector, $[\mathbf{M}]$ is the diagonal mass matrix, $[\mathbf{C}]$ is the damping matrix and $\mathbf{F}(t)$ is the vector of external forces on particles. The motion 
equations of the particle system are solved through an explicit central finite differences scheme. The calculation cycle is illustrated in Figure 1(b). The details of the implementation and verification of DLSM can be found in these paper [24-25].

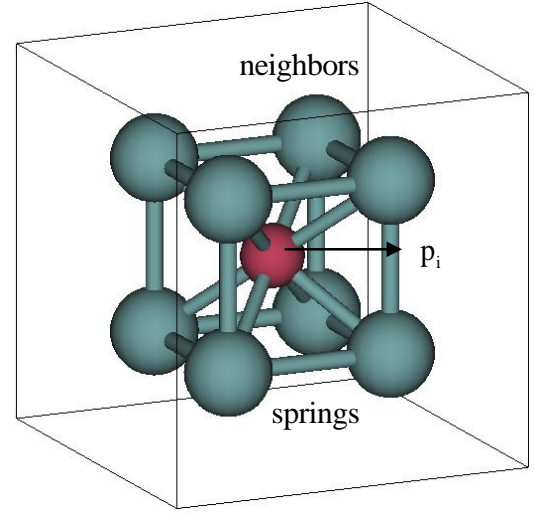

(a) The physical model of DLSM

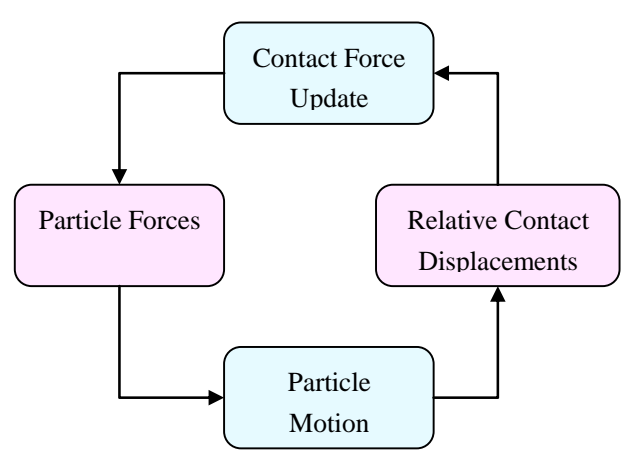

(b) Calculation cycle

Figure 1. The physical model and the calculation cycle of DLSM.

Due to the explicit considerations of the discontinuous microstructure of material, the model has advantages in modelling of material failure behaviour. For example, Kazerani, et al., (2010) used DLSM to model dynamic cracking propagation of PMMA by implementation of a rate-dependent cohesive law [6], and Zhu et al., (2011) applied the DLSM on modelling wave propagation through jointed rock masses [27]. Moreover, the DLSM is also used in analysis of the cutting process of coal under a single blade [26], and dynamic fracture toughness of granite [25]. In DLSM, there is no need to form the global stiffness matrix and only a local interaction is considered during calculation. This is very suitable for large scale parallel computing implementation [25]. The DLSM can be viewed as a mesh-less method like EFG and FPM, but more closer to DEM methods. Compared with the particle-based DEM, the DLSM can directly use macroscopic parameters without a calibration process. This is regarded as the main advantage over other discrete element based methods. Moreover, DLSM also have advantages over existing mesh-less methods, e.g. EFG, FPM and SPH, on stability, no integration requirement and simplicity in dealing with heterogeneity problems. Due to the mesh-less and natural discrete properties of DLSM, it is suitable for dynamic discontinuous computations.

\subsection{Desiccation cracking of fine-grained soils}

In order to clarify the mechanism of desiccation induced shrinking and cracking in fine-grained soils, a series of experimental tests with three different fine-grained materials: Bioley silt, Sion silt and La frasse clay were performed by Peron, et al., [16]. In this paper, Bioley silt is adopted as experimental result to compare with numerical simulation by DLSM and its physical property is shown in Table 1. The tests comprised three types: free desiccation, constrained desiccation(Figure 2) and crack pattern tests (Figure 5). For the free desiccation test, soil slurry was poured into a mould with dimension: $295 \mathrm{~mm} \times 49 \mathrm{~mm} \times 12$ 
$\mathrm{mm}$, and put on a Teflon plate to minimize friction. The environment (climate chamber) of the test was temperature and humidity controlled. During drying, the strain evolution with water content was measured as shown in Figure 3. For one dimensional constrained drying test, the sample size was same as that of free drying ones. However, the drying was proceeded on notched base(see Figure 2). Its strain evolution with water content was illustrated as in Figure 4. Crack pattern test was same as the other two tests, but the dimension is $300 \mathrm{~mm} \times$ $300 \mathrm{~mm} \times 4 \mathrm{~mm}$ and $300 \mathrm{~mm} \times 300 \mathrm{~mm} \times 12 \mathrm{~mm}$. The final crack pattern was as shown in Figure 5 .

Table 1. Physical properties of Bioley clayey silt.

\begin{tabular}{cccc|c|c|}
\hline Liquid limit & Plastic limit & Unit weight of solid particles & Clay fraction \\
\hline $31.8 \%$ & $16.9 \%$ & $27.1 \mathrm{KN} / \mathrm{m} 3$ & $27 \%$ \\
\hline & & & & \\
\hline & & & & & \\
\hline
\end{tabular}

Figure 2. Results of one dimensional constraint drying tests, [16].

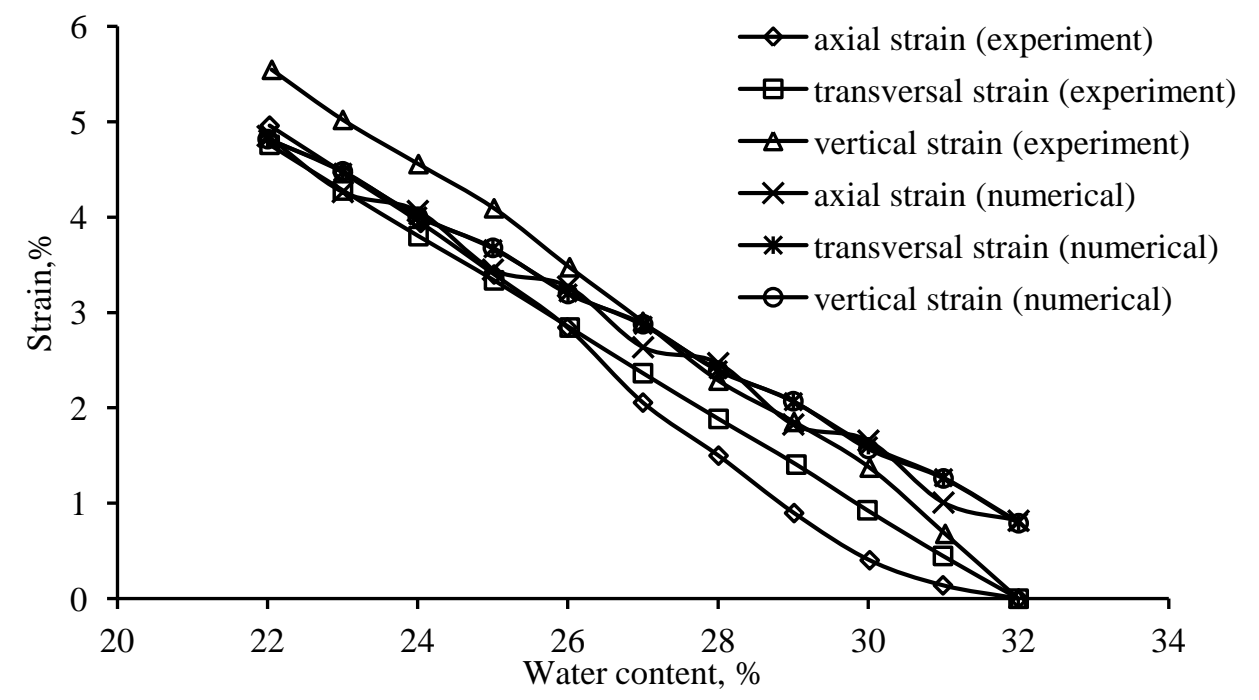

Figure 3. Evolution of strains with respect to water content for free drying shrinkage experimental result, adopted from [15] and numerical prediction. 


\subsection{Drying shrinkage in DLSM}

For free drying shrinkage, the volumetric deformation of the model can be given as Peron et al., (2009b) [15]:

$$
\varepsilon_{V}=1-\exp \left(-\alpha \frac{t}{\tau}\right)
$$

where $\alpha$ is a material parameter ( $\alpha>0)$, and $\tau$ is the total duration of the experiment $(0 \leq t \leq \tau)$. When $\alpha$ is small, the above equation can be replaced by a linear relationship as:

$$
\varepsilon_{V}=\left(-\beta \frac{t}{\tau}\right)
$$

where $\beta$ is a material parameter $(\beta>0)$, this linear relationship has also been proven in the experiment by Peron et al., 2009a [15] . In DLSM, assume a homogeneous deformation applied on the numerical model, and then the induced force in the spring network will be:

$$
F=-\beta\left(\frac{t}{\tau}\right) l k_{n}
$$

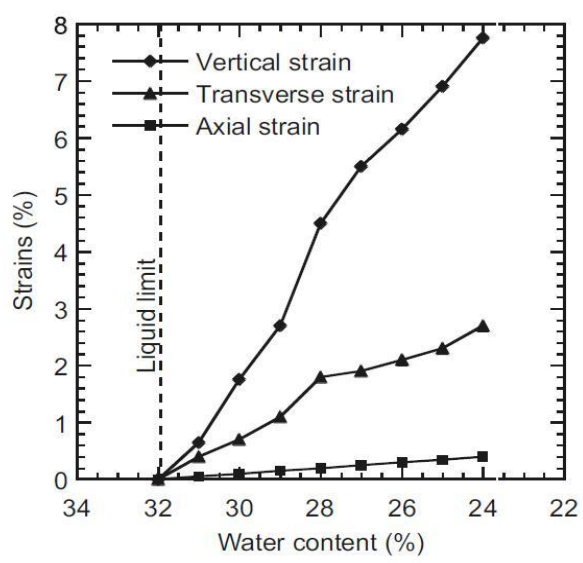

Figure 4. Strain evolution with water content for one dimensional constraint drying test, [16]

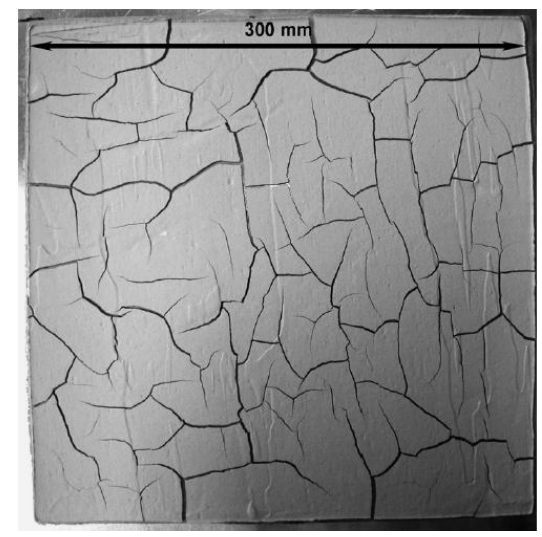

Figure 5. Final crack pattern in crack pattern test [16]. 
In order to produce the given deformation, the drying shrinkage is simulated by introducing a suction force term into the DLSM model. The suction force $\left(F^{w}\right)$ should be:

$$
F^{w}=-F=\beta\left(\frac{t}{\tau}\right) l k_{n}
$$

here, the parameter $\beta$ is actually the volume strain at the end of the test. A combined component spring bond $\left(F^{s}\right)$ and water bond $\left(F^{w}\right)$ is introduced as shown in Figure 6 (a), the total forces are calculated as:

$$
\begin{gathered}
F^{n^{\prime}}=\beta\left(\frac{t}{\tau}\right) l k_{n}+u_{n} k_{n} . \\
F^{s}=u_{n} k_{n} .
\end{gathered}
$$

in which $F^{n}$ is total force and $F^{s}$ is spring force due to spring deformation. As shown in Fig. 6 (b), when the air enters into the soil, the suction force will drop to zero. The criterion for air entrance is given as

$$
\begin{gathered}
F^{n^{\prime}} \geq F^{\alpha^{*}} . \\
\beta\left(\frac{t}{\tau}\right) l k_{n}+u_{n} k_{n} \geq F^{\alpha^{*}} .
\end{gathered}
$$
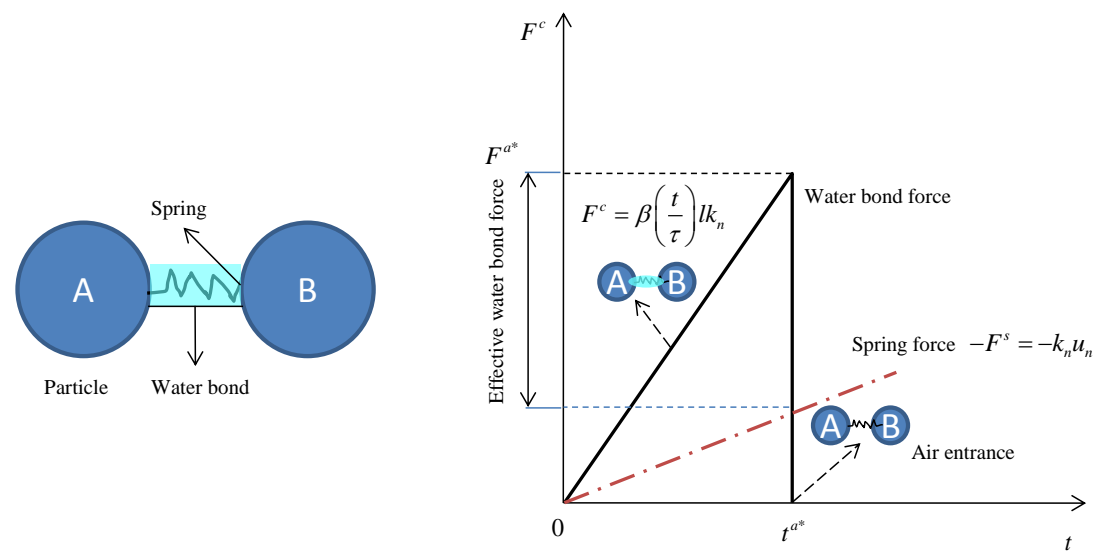

Figure 6. Illustration of water bond force (a) and its constitutive model (b)

Assume that there is a maximum shrinkage stress as $\sigma_{\mathrm{s}}^{*}$. Then, the ultimate water bond deformation can be determined from following equation:

$$
u^{w^{*}}=\frac{\sigma_{s}^{*}}{E} d
$$

in above equation, $d$ is the distance of the two particles connected by spring and water bonds. The bond failure for springs will be determined from 


$$
u^{w^{*}}=\frac{\sigma_{t}^{*}}{E} d .
$$

here, $\sigma_{t}^{*}$ the macro tensile strength of the unsaturated soil. All parameters needed in DLSM can be determined from the corresponding macro parameters. The next is how to introduce the concept of heterogeneity in this simulation. In [15], the heterogeneity was represented by the different particle size in the DEM model. However, this (different particle size) is, intrinsically, different from the physical state of fine-grained soils where the structure heterogeneity is not obvious. In this numerical investigation, another heterogeneity method which has been widely used by FEM to simulate the fracturing process is utilized. It is achieved by randomly assigning mechanical parameters of springs with different values. In this model, mechanical deformation of soil is treated as homogeneity. When all the water bond failure (air entry) is identical, there will be no tensile deformation formed and then no soil crack, as illustrated in Figure 7 (c). If the water bond failure is heterogeneity(Figure 7 (d)), the tensile deformation can be formed in the spring and then mechanical failure (crack) will be induced. If we use a coarse resolution particle, the REV size, the particle of fine-grained soil (around 0.5um) compared with the resolution of DEM particles $(1 \mathrm{~mm})$, should be treated as homogeneity. This hypothesis can be strongly supported by following experimental evidences:

1. The experimental results showed that soil never cracked without air entry. In this model, there are two levels of failure, water bond failure and spring bond failure. The former one induces tensile deformation on the spring bond, thereby leading to mechanical failure and forming final crack.

2. The water bond failure criteria are based on an effective water bond deformation. When the movement of the water bond is constrained, the value of effective water bond deformation increases and easily reaches the failure critical deformation. This addresses crack initiating and propagating in constrained desiccation. However, in free shrinkage, no matter how high the negative pore water pressure (suction) is, there is no induced crack as in free desiccation, the effective water bond deformation is zero and the water bond cannot break. Therefore, the spring bond cannot damage, and the final result is no desiccation crack. In this sense, the water bond performs as an either protector or trigger for the soil crack dependent on constraint condition.

3. Air entering into pore induces tensile deformation in the air entering zone as the water bond in neighbouring zone keeps shrinking, then the crack will happen at these air entering zones. The heterogeneity of the air entry time for each point is also essential for forming these cracks. So, there are two important issues for soil cracks: air entry and heterogeneity.

In the DLSM, these factors are fully coupled. In following section, the numerical modelling study on soil desiccation will be conducted by using the DLSM with the constitutive law for the water bond force.

To determine the parameter $\alpha$, one considers that at the end of the test $\varepsilon_{V}$ takes the value $\varepsilon_{V}^{f}$ at $t=\tau$.This is suitable for homogeneous drying shrinkage only; the heterogeneous 
shrinkage (directly related to a heterogeneous water content distribution) can be simulated by imposing a dependency between the parameters $\alpha$ and the spatial position of the grains.

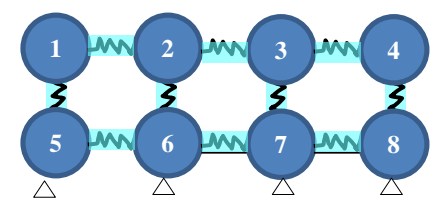

(a) Initial state

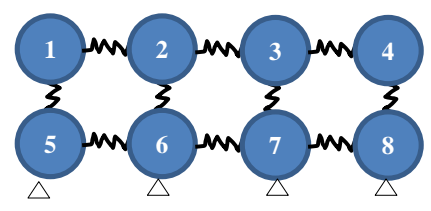

(c) Homogeneity water bond failure

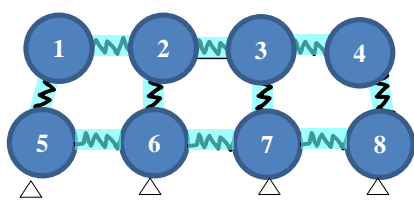

(b) Shrinkage deformation

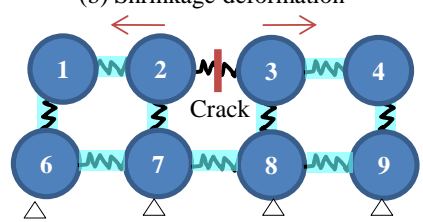

(d) Heterogeneity water bond failure

Figure 7. Illustration of the forming of soil crack

\section{MODELLING DESICCATION CRACKING}

\subsection{Parameter calibration}

The parameter determination is performed on the result of free desiccation tests of [16]. In order to calibrate the parameter $\alpha$, Equation (5) was used. This allows the determination of $\varepsilon_{V}$ the value of volumetric strain at the end of the simulation for $w=w_{f}=22 \%$ and $t=\tau$, see from Figure 3 . The $\varepsilon_{V}$ is 0.145 . For the free desiccation, logitudial direction was considered, so the parameter $\alpha$ can be expressed

$$
\alpha=-\ln \left(1-\frac{1}{3} \varepsilon_{V}\right)
$$

Then the value of $\alpha$ is 0.0483 . With the value of $\alpha$, the whole free drying shrinkage process was performed (Figure 8). The predicted strain respect to water content is shown in Figure 3 and they have good agreement.

\subsection{Constrained desiccation}

For constrained desiccation, the parameter cillibrated from free shrinkage simulation are adopted to reproduce crack parttern in linear desccation test of [16] using the proposed DLSM model. The result is shown in Figure 9. Basically, the number of cracks depends on the random ratio (i. e. degree of heterogeneity) and particle size and this will be discussed in section 4 . The first crack was not initiated at the middle of the soil bar, which is different from the prediction of Kodikara and Choi (2006) [8]. In Kodikara and Choi 2006, the first crack was expected to initiate at the middle of a soil bar as the tensile stress there was the largest one. The simulation result is similar to the test results. 

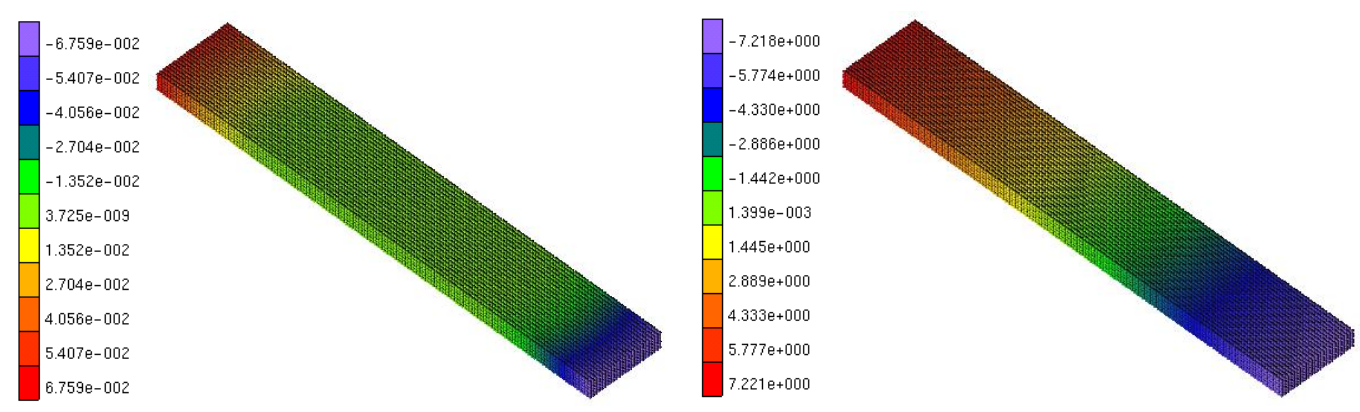

Figure 8. The free shrinking, step 1 (a) and step 30 (b).
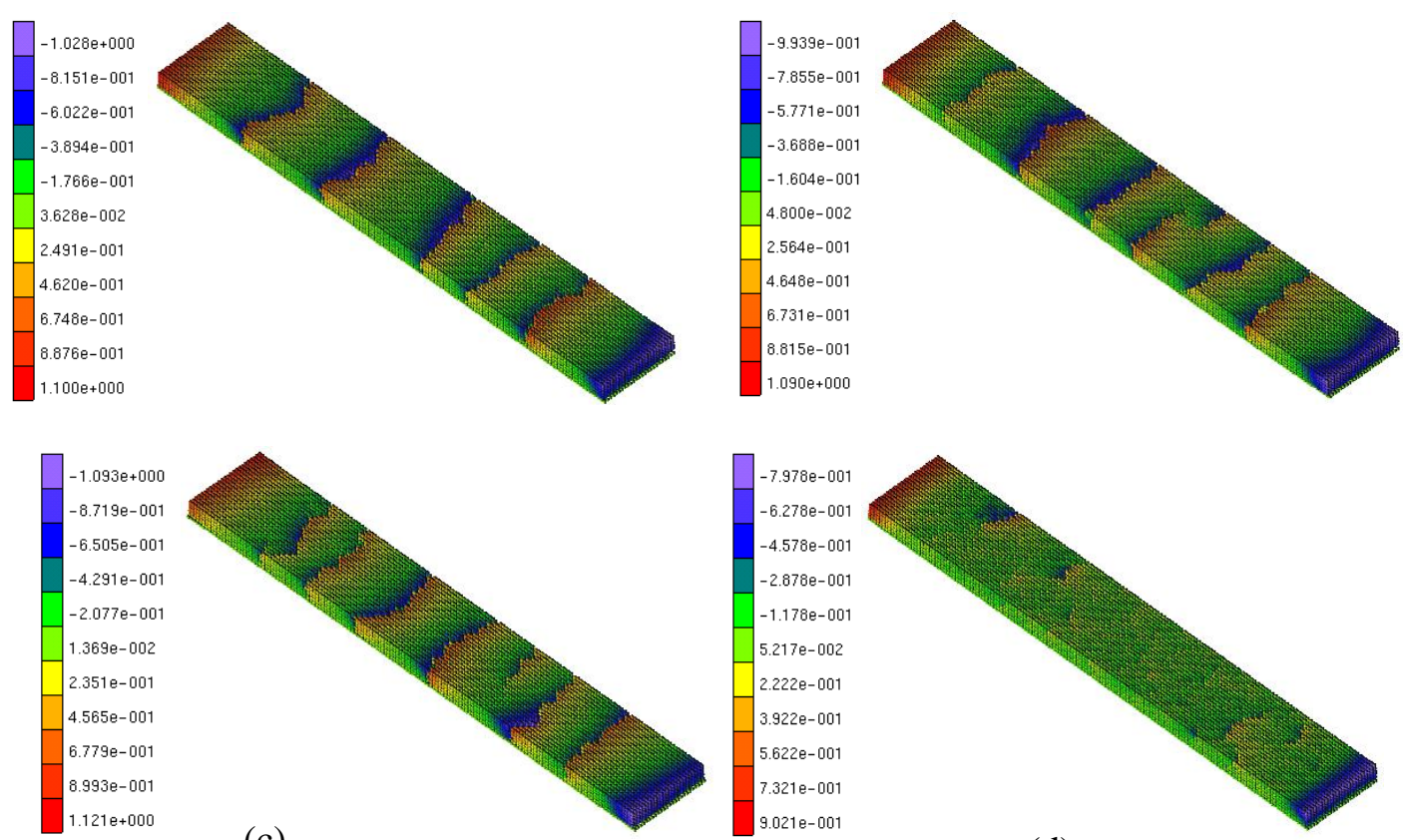

(d)

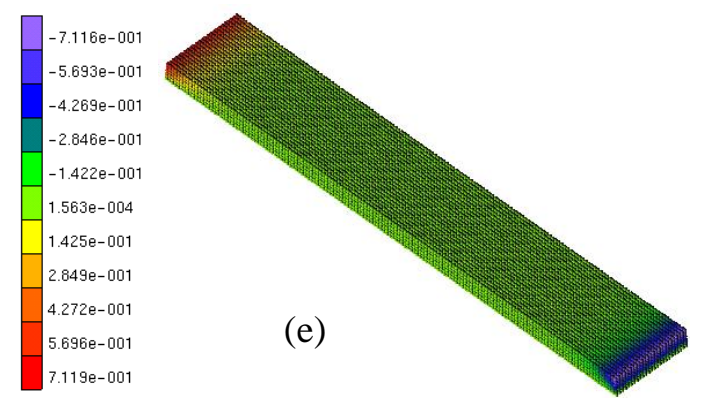

Figure 9. Shrinkage strain of 2D constrained modelling crack in soil bar (constrained drying) for random ratio $5 \%$ (a), $10 \%$ (b), 30\% (c) and 50\% (d); drying without air entry difference, random ratio is zero $(\mathrm{e})$.

\subsection{The 3D crack pattern simulation}

In the crack pattern simulations, cracks occurred in all four times of modelling (see Figure 10). Generally, crack initiates at one corner of the mould and then propagates. It is 
worth to be noted that different cracks always intersect with each other by approximate right angle. This is close to the observation result of [10]. This is due to the reason that tensile stress built in the direction perpendicular to the current crack paths is the largest.

\section{DISCUSSION}

\subsection{Influence of random ratio}

Random ratio adopted here is the percentage of water bonds which have lower effective water bond force(see Figure 6 (b)). For example, if the random ratio is 5\%, that is $5 \%$ of the particles' water bonds in the soil sample are easier to get cracked compared with other water bonds. As shown in Figure. 9. desiccation crack pattern is closely related with the degree of heterogeneity of water bond, namely, the prescribed random ratio for drying process. Generally, higher the random ratio is, more and smaller the observed cracks there are. When the random ratio selected reaches 50\%, all the cracks become smaller and more complicated. If all the water bonds are distributed homogeneously, no cracks happen at all (Figure. 9(e)).
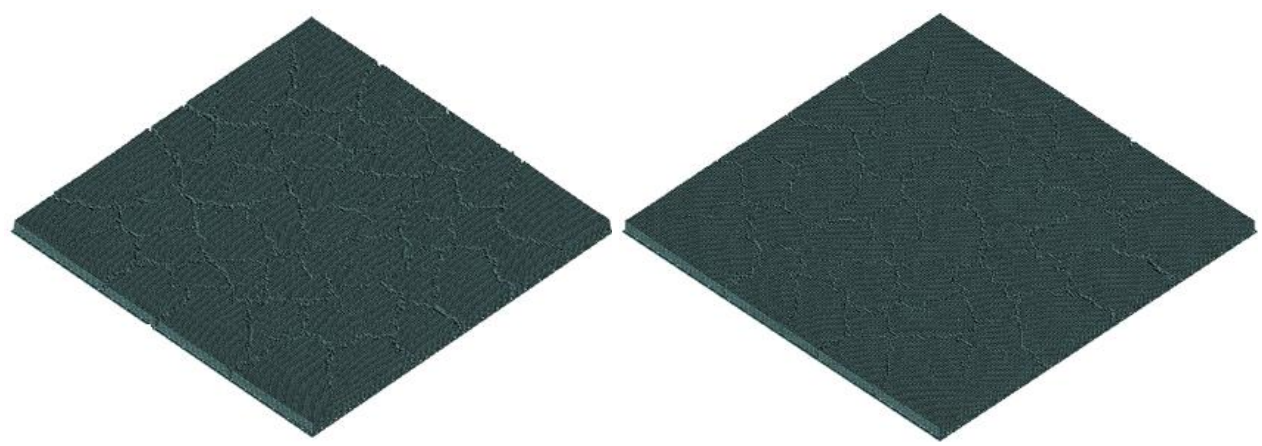

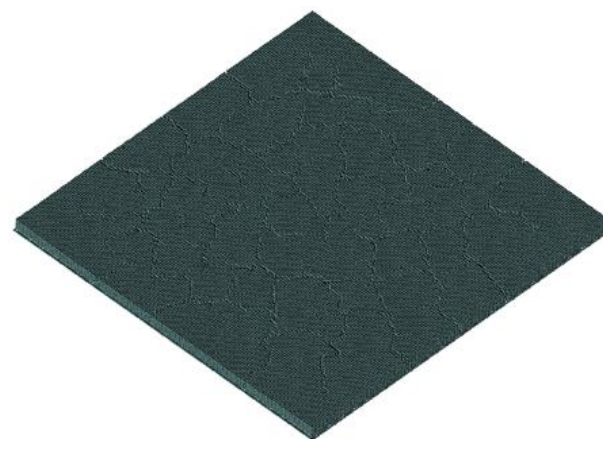

(c)

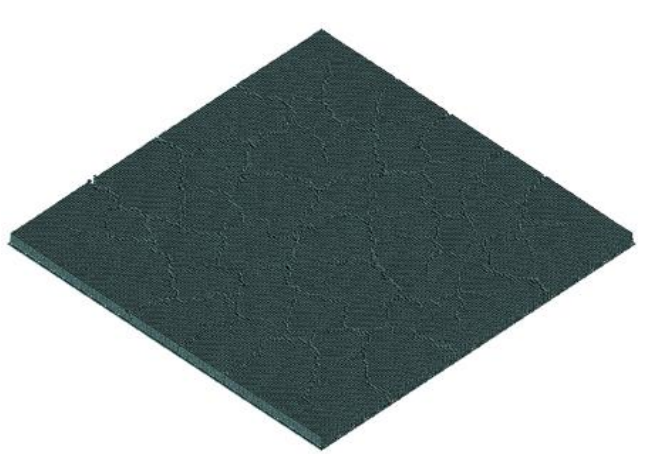

(d)

Figure 10. The 3D crack pattern simulation, four times calculation with same random ratio $(10 \%)$ 


\subsection{Influence of particle size}

Particle size is another factor affecting desiccation crack pattern in soil. Figure. 11 illustrates the influence of particle size on crack pattern. From Figure. 11, it is easy to observe that soil made of smaller particles has more potential to get cracked. This can be validated by experimental results $[11,19]$. Crack usually initiated and propagate in fine-grained materials including clayey soils [16], gels [19], fine-grained mining wastes or tailings [18]. Yesiller et al., (2000) proposed that "large amount of cracking were always observed in samples with high fine contents and high suction in soils, and less cracking in soil with low fines content"[23]. It is obvious that porous materials with finer particles have smaller pore size.
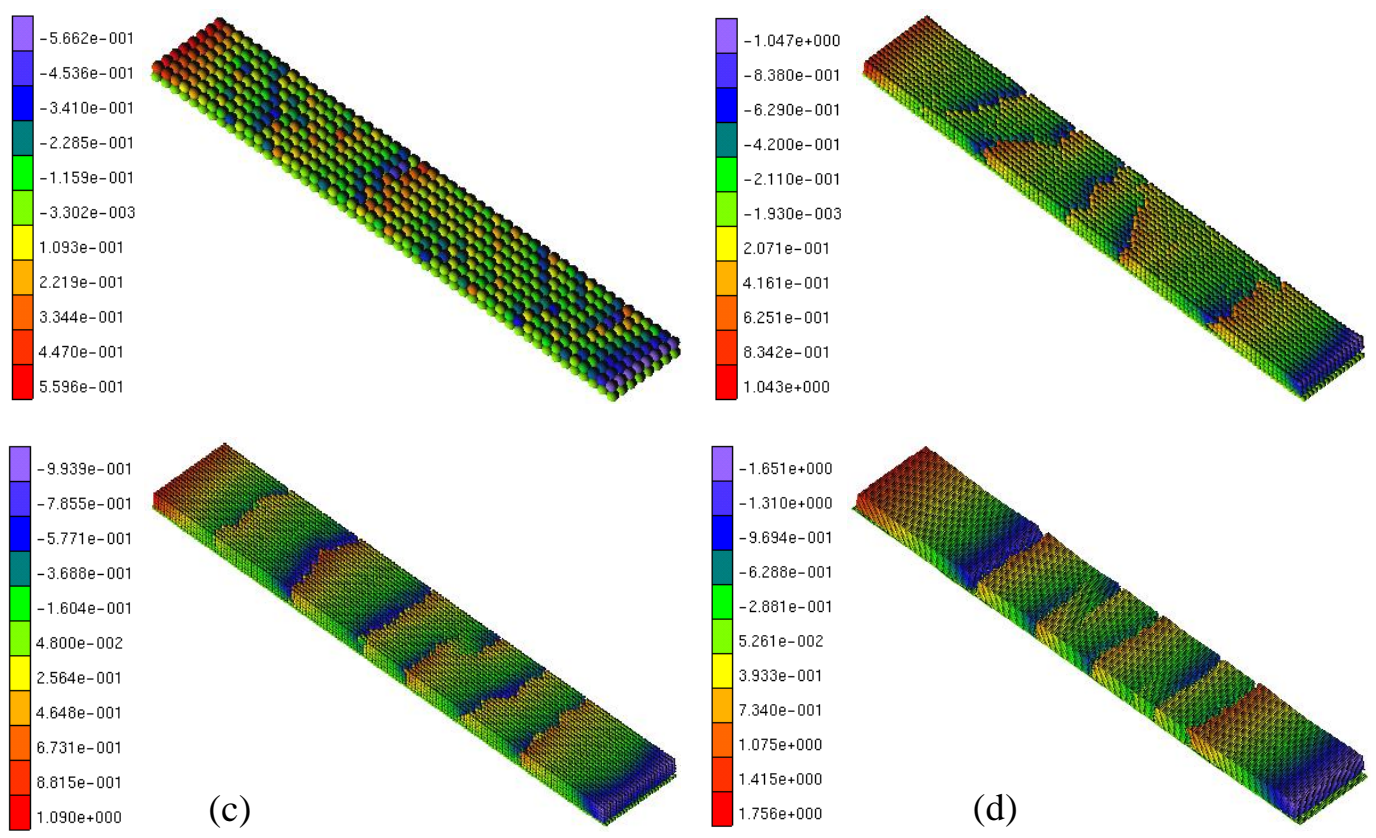

Figure 11. Desiccation induced cracks for different particle size, $6 \mathrm{~mm}$ (a) , $3 \mathrm{~mm}$ (b), $2 \mathrm{~mm}$ (c) and $1 \mathrm{~mm}(\mathrm{~d})$.

\section{CONCLUSIONS}

Numerical simulation of drying shrinkage and cracking in fine-grained soils, with the help of DLSM and proposed suction force constitutive law, is presented. The results of the numerical modelling agree with the experimental tests observations well. Apart from that, on micro mechanics level, some factors like heterogeneity or random ratio and particle size are discussed. It is found that they have considerable influence on crack pattern formation.

\section{REFERENCES}

[1] Bazant, Z.P., Tabbara, M.R. Kazemi, M.T., and Cabot, G.P., "Random particle model for fracture of aggregate or fiber composites". J. Eng. Mech, 116(8), 1686-1705, 2009. 
[2] Buxton, G.A., Care, C.M. and Cleaver, D.J., "A lattice spring model of heterogeneous materials with plasticity". Modelling Simul. Mater. Sci. Eng., 9(6), 485-497, 2001.

[3] Deng, G., Shen, Z.-J., and Yang, D.-Q., Numerical simulation of crack formation due to desiccation in clay surface. Chinese J. of Geotech. Eng., 28, 241-248, 2006

[4] Dixon, D., Chandler, J., Graham, J., and Gray, M.N., "Two large-scale sealing tests conducted at Atomic Energy of Canada's underground research laboratory: The buffercontainer experiment and the isothermal test". Can. Geotech. J., 39(3), 503-518, 2002.

[5] Hallett, P.D and Newson, T.A., "Describing soil crack formation using elastic-plastic fracture mechanics". Euro. J. Soil Sci., 56(1):31-38, 2005.

[6] Kazerani, T., Zhao, G.F., and Zhao, J., "Dynamic fracturing simulation of brittle material using the distinct lattice spring method with a full rate-dependent cohesive law". Rock Mechanics and Rock Engineering, 43(6), 717-726, 2010.

[7] Kodikara, J.K., Rahman, F., and Barbour, S.L. "Towards a more rational approach to chemical compatibility testing of clay". Can. Geotech. J., 39(3), 597-607, 2002.

[8] Kodikara, J.K. and Choi., X. A simplified analytical model for desiccation cracking of clay layers in laboratory tests. in 4th International Conference on Unsaturated Soils, April 2, 2006 - April 5, 2006. 2006. Carefree, AZ, United states: American Society of Civil Engineers.

[9] Konrad, J.M. and Ayad, R., "An idealized framework for the analysis of cohesive soils undergoing desiccation". Can. Geotech. J. 34(4), 477-488, 1997.

[10] Morris, P.H., Graham, J., and Williams, D.J. "Cracking in drying soils". Can. Geotech. J. 29(2), 263-277, 1992.

[11] Nahlawi, H. and Kodikara, J.K. "Laboratory experiments on desiccation cracking of thin soil layers". Geotech. Geol. Eng., 24(6),1641-1664, 2006.

[12] Nahlawi, H., Chakrabarti, S., and Kodikara, J., "A direct tensile strength testing method for unsaturated geomaterials". Geotech Test. J.. 27(4),356-361, 2004.

[13] Ostoja-Starzewski, M., "Lattice models in micromechanics". Appl. Mech. Rev. 55(1),3559, 2002. 
[14] Ostoja-Starzewski, M., Sheng, P.Y. and Jasiuk, I. "Damage patterns and constitutive response of random matrix-inclusion composites". Eng. Frac. Mech. 58(5-6), 581-606, 1997.

[15] Peron, H., Delenne, J.Y. Laloui, L., and Youssoufi. M.S.El., "Discrete element modelling of drying shrinkage and cracking of soils". Comput. Geotech., 36:61-69, 2009

[16] Peron, H., Hueckel, T., Laloui, L., and Hu, L.B., "Fundamentals of desiccation cracking of fine-grained soils: Experimental characterisation and mechanisms identification". Can. Geotech.J., 46(10), 1177-1201, 2009.

[17] Rayhani, M.H.T., Yanful, E.K., and Fakher, A., "Physical modelling of desiccation cracking in plastic soils". Engi. Geol., 97,25-31, 2008.

[18] Rodriquez, R., Sánchez, M., Ledesma, A., and Lloret, A., "Experimental and numerical analysis of desiccation of a mining waste". Can. Geotech. J., 44, 644-658, 2007.

[19] Scherer, G.W. "Crack-tip stress in gels". J. Non-Cryst. Sol., 144: 210-216, 1992.

[20] Shen, Z.-J. and Deng, G., "Numerical simulation of crack evolution in clay during drying and wetting cycle". Rock and Soil Mech., 25, 1-6+12, 2004.

[21] Sun, J., Wang, G. and Sun, Q., "Crack spacing of unsaturated soils in the critical state". Chinese Science Bulletin, 54(12),2008-2012, 2009.

[22] Towner, G.D., "The mechanics of cracking of drying clay". J. Agri. Eng. Res., 36(2),115124, 1987.

[23] Yesiller, N., Miller, C.J. Inci, G. and Yaldo, K., "Desiccation and cracking behavior of three compacted landfill liner soils". Eng. Geol., 57,105-121, 2000.

[24] Zhao, G.-F., Fang, J. and Zhao, J. "A 3D distinct lattice spring model for elasticity and dynamic failure". Int. J. Numer. Anal. Meth. Geomech., 35,859-885, 2011.

[25] Zhao, G.F. "Development of Micro-macro Continuum-discontinuum Coupled Numerical Method. PhD thesis". École Polytechnique Fédérale de Lausanne (EPFL), Lausanne, Switzerland. 2010.

[26] Zhao G.F, Zhao J. "Microscopic numerical modelling of the dynamic strength of brittle rock". In: Ma GW, Zhou YX, editors. Analysis of discontinuous deformation: new developments and applications. Singapore: Research Publishing. 633 - 640, 2009. 
[27] Zhu, J.B., Zhao, G.F. Zhao, X.B. and Zhao, J. "Validation study of the distinct lattice spring model (DLSM) on P-wave propagation across multiple parallel joints". Comput. Geotech., 38(2),298-304, 2011. 